\title{
Optimal Number of Plants: Trade-off between Production and Transportation Costs
}

\section{INTRODUCTION}

OVERTHE past several years the concept of minimizing total costs has received considerable attention in the literature on logistical management $[2,3,4]$. It is argued that in designing a logistics system, minimizing only transportation costs leads to sub-optimal results because of the interaction between transportation and other distribution costs. It is suggested that a better approach is to minimize the total costs which are usually defined to include transportation and inventory costs. This literature considers the production system. in particular the plant configuration, as being given.

The next logical step in terms of really minimizing costs would be to define total costs to include production as well as distribution costs and not to consider the plant configuration as a given. A firm should design the plant configuration so as to minimize the sum of production and distribution costs. Unfortunately, the literature on production-distribution systems is very small. Pomper [5] takes into account both production and distribution costs in the context of a complex capacity expansion decision.

The objective of this paper is to analyze the trade-off between economies of scale in production and the cost of transporting the product to the customers. A firm can minimize its transportation costs by operating a very large number of plants. However. this will tend to increase the production costs. If there are economies of scale in production. then the production costs can be minimized by having only one plant. There must exist an optimal number of plants such that the sum of production and transportation costs is minimized.

\section{THE MODEL}

We formulate a model to analyze the trade-off between production economies of scale and transportation costs. In order to focus only on this particular issue. it is assumed that the availability and costs of factor inputs (i.e. raw materials, labour, energy) do not affect the decision on plant configuration. It is also assumed that distribution costs consist only of transportation costs.

Let the quantity demanded in physical units for the firm's product be $D$ and assume that this quantity is uniformly distributed over area $A$. The firm has to decide on the number of plants to operate, denoted by nt. and on the production volume of each plant. denoted by $d_{i}, i=1,2, \ldots n$. Each plant has to distribute its output, $d_{i}$, to customers uniformly spread over area $\left(d_{i i} D\right) . A$, so that the total sum of $d_{i}$ equals $D$.

To calculate the transportation costs for each plant, we make the simplifying assumption that each plant is located at the center of a circular region of area $\left(d_{i} / D\right) A$ which it supplies. This will obviously lead to underestimating the transportation costs. and hence result in underestimating the optimal number of plants. Still. the model does yield some useful results.
The cost of shipping one unit of the product over distance $x$ is assumed to be given by $c^{\prime \prime}$. Whers $c$ is a cost parameter. Transportation costs as a function of distance range from being completely insensitive to distance (that is. $\beta=0$ 1 to varying directly with distance (that is. $\beta=1$ ). However, most cases lie between these extremes [1. ch. 6]. Thus the parameter $\beta$ captures the economies of scale with respect to distance in shipping.

Let the shipping radius of a plant be $r_{i}$ : then. we have

$$
\pi r_{i}^{2}=\frac{d_{i}}{D} \cdot t .
$$

To obtain the transportation cost for a plant. consider a circular ring of thickness $i x$ and radius $x$ with the plant at the center. The quantity demanded in this ring is $2 \pi x \times(D$ A ) and the cost of transporting this quantity is $2 \pi x+x(D / A)\left(x^{b}\right.$. Integrating this cost over $x$ from 0 to $r_{i}$, we obtain the transportation cost for plant $i$ to be

$$
T C_{i}=\frac{2 c}{(2+\beta)}\left(\frac{A}{\pi D}\right)^{\beta 2}\left(d_{i}\right)^{1+\beta}=
$$

The production cost for plant $i$ is assumed to be given by

$$
P C_{i}=a d_{i}^{x}, \quad x \leqslant 1
$$

where the parameter $x$ captures the economies of scale effect in production

The optimization problem for the firm is to choosen and $d_{i}$ (where $d_{i} \geqslant 0$ ) so as to

$$
\min C=\sum_{i=1}^{n}\left(P C_{i}+T C_{i}\right) . \quad i=1.2 \ldots \ldots n .
$$

As formulated. problem (3) is intractable. One way to reformulate it is

$$
\min C=\sum_{i=1}^{s}\left(P C_{i}+T C_{i}\right) . \quad i=1,2 \ldots . . .
$$

where $N$ is some arbitrarily large number. Then the optimal number of plants, $n^{*}$. is equal to the number of elements in the set ${ }_{i} i d_{i}^{*}>0 ;$, where $d_{i}^{*}$ is the optimal solution to problem (4).

If one writes down the Kuhn-Tucker conditions for problem (4). by visual inspection it can be seen that the optimal solution is of the form

$$
d_{i}^{*}= \begin{cases}(1, M) D, & \text { for } i=1,2 \ldots \ldots, W \\ 0 . & \text { for } i>M\end{cases}
$$

where $M$ is some integer number less than $X$. That is, the optimal solution to problem (3) is such that all the plants produce an equal amount. but we still do not know the optimal number of plants.

Now, for the time being. assume that the number of plants need not be an integer. Using the above result and equations (1) and (2) problem (3) can be reformulated as

$$
\min _{n \rightarrow 0} C(n)=n\left[\frac{2 \cdot D}{(2+\beta)}\left(\frac{A}{\pi}\right)^{\beta 2} n^{-1-\beta 2}+A D^{2} n^{-2}\right] \text {. }
$$


Differentiating the above equation and equating to zero gives the optimal solution

$$
n^{*}=\left[\frac{\beta\left(D^{1-x} A^{y z}\right.}{(2+\beta)(1-x) \pi^{32}}\right],
$$

where

$$
\dddot{i}=\frac{1}{1+(\beta 2)-x} .
$$

It is easy to show that $n^{*}$ satisfies the second-order optimality condition. and hence is a local minimum. Furthermore. it can be shown that the function $C(n)$ in equation (5) has only one local minimum. This implies that $n^{*}$ is the global minimum. and that the optimal integer solution to problem (5) is equal to one of the two closest integers to $n^{*}$.

\section{INTERPRETATION}

Given the nature of the analysis presented here, the value of this model lies not in determining precisely the optimal number of plants, but rather in yielding some insight into how the trade-off between production and transportation costs affects the optimal number of plants.

It is useful to rewrite equation (6) in a simpler form as follows. Define $P C(n)$ and $T C(n)$ as the total production and transportation costs for the firm if it operates $n$ plants of equal size. Also let $R(n)=T C(n) / P C(n)$. Using equations (1) and (2) to derive the value of $R(1)$ and substituting it into equation (6). we have

$$
n^{*}=\left[R(1) \frac{\beta}{2(1-x)}\right] \text {. }
$$

The ratio $R(1)$ is equal to the ratio of transportation costs to production costs if the tirm decides to operate only one plant. As expected intuitively. the optimal number of plants increases as this ratio increases.

Using equations (1), (2) and (7) and doing some algebraic simplification, we have

$$
R\left(n^{*}\right) \equiv \frac{T C\left(n^{*}\right)}{P C\left(n^{*}\right)}=\frac{2(1-x)}{\beta} .
$$

Therefore, at the optimum plant configuration, the ratio of a firm's transportation costs to production costs is equal to $2(1-x) / \beta$. As $x$ decreases, that is, as production economies of scale increase. the ratio $R\left(n^{*}\right)$ increases. As $z$ decreases. the firm makes the optimal decision to build fewer plants. thus decreasing its production costs and increasing its transportation costs.

As $\beta$ decreases, the optimal ratio $R\left(n^{*}\right)$ increases. That is, as economies of scale in transportation increase. the firm's transportation costs increase as a fraction of its production costs. This might seem counterintuitive. until it is realized that as $\beta$ decreases, shipping the product over long distances becomes relatively less expensive: hence. the firm's optimal decision would be to build fewer plants to take advantage of production economies of scale. Therefore. economies of scale in transportation facilitate exploiting production economies of scale.

Equation (8) can also be used as a diagnostic check. If the actual ratio of transportation costs to production costs for a firm with its existing plant configuration is greater than $R\left(n^{*}\right)$, then the firm is probably spending too much on transportation and might want to consider building an additional plant. Conversely, if its actual ratio of transportation to production costs is less than $R\left(n^{*}\right)$. then the company is probably not fully exploiting the production economies of scale, and should consider reducing the number of plants it operates.

\section{REFERENCES}

1. Ballou RH (1973) Business Logistics Management. Prentice-Hall, Englewood Cliffs. New Jersey.

2. Bowersox DJ (1978) Logistical .Management, 2nd edn. Macmillan. New York.

3. Flak'S M (1963) Total cost approach to physical distribution. Bus. Mgmt 24, pp. 55-61.

4. LeKashiman R \& Stolle JF (1965) The total cost approach to distribution. Bus. Horizons 44, pp. 33-46.

5. POMPER CL (1976) International Investment Planning: an integrated approach. North-Holland, Amsterdam.

Aneel Karnani

Graduate School of Business

(January 1982)
Administration

The University of Michigan

Ann Arbor

MI 48109

USA 